\title{
XXVI. Dolerophanit als Hüttenproduct.
}

\author{
Von
}

\author{
J. E. Strandmark in Lund.
}

(Mit 3 Textfiguren.)

Vor einigen Jahren wurde dem geologisch-mineralogischen Institute in Lund durch den Director des Kupferbergwerkes Åtvidaberg in Östergötland, Dr. C. Hudelius, eine als Hüttenproduct gebildete Substanz zugesandt. Bei Åtvidaberg werden kiesige Kupfererze in grossem Maassstabe verhüttet; Cementkupfer wird auf dem mit feuerfestem Thon ausgekleideten und mit einem Gemenge von Quarz und etwas Schlacke ausgeschlagenen Herde eines Flammenofens eingeschmolzen. Auf den durch das abwechselnde Erhitzen und Erkalten in dieser Sohle entstandenen Spalten war die oben erwähnte Substanz in kleinen, braunen, ziemlich stark glänzenden Krystallen gebildet worden. Diese erwiesen sich bei einer chemischen Untersuchung als basisches Kupfersulfat von der Zusammensetzung 2 $\mathrm{CuO} . \mathrm{SO}_{3}$. Die Analyse gab nämlich:

$$
\begin{aligned}
& \mathrm{CuO} \quad 65,95 \% \text { berechnet: } 66,50 \% \\
& \mathrm{SO}_{3} \frac{34,43}{100,38 \%}-\frac{33,50}{100,00 \%}
\end{aligned}
$$

Die Substanz wurde vor der Analyse bei $+108^{\circ} \mathrm{C}$. getrocknet. Das Kupfer wurde aus der Lösung ais Sulfid gefällt und nach Reduction im Wasserstoffstrome als Sulfür gewogen. Die Schwefelsäure wurde in einer anderen Probe auf gewöhnliche Weise bestimmt.

Beim Glühen im Gebläse entgeht der Substanz die Schwefelsäure; so wurde bei einem Versuche 66,46\% Kupferoxyd als Rest erhalten.

Ein basisches wasserfreies Kupfersulfat von derselben Zsammensetzung und - wie aus den Winkelmessungen hervorging - auch von derselben Krystallform ist von A. Scacchi als Mineral gefunden und mit dem Namen Dolerophanit belegt worden. Dasselbe war bei der Eruption des Vesuvs im Oclober 1868 als pneumatolytisches Product entstanden und ist von 
Scacchi in krystallographischer Beziehung ausführlich untersucht worden 1). Von dessen physikalischen und optischen Verhältnissen meldet dieser Forscher jedoch nichts.

Die Aufstellung Scacchi's wurde von V. Goldschmidt derart geïndert, dass man in dem Axenverhältnisse des Dolerophanits ${ }^{2}$ ) eine Analogie mit dem "wahrscheinlich isomorphen " Lanarkit ${ }^{3}$ ), dem entsprechenden Bleisulfate $2 \mathrm{PbO} . \mathrm{SO}_{3}$, finden könne. Die Formen der beiden Mineralien liessen sich jedoch nicht zur Uebereinstimmung bringen, und Goldschmidt hat daher auch später dem Dolerophanit cine selbständige, natürlichere Aufstellung gegeben ${ }^{4}$ ).

Die Dolerophanitkrystalle von Åtvidaberg sassen, wie oben erwähnt, an den Spaltwänden des Ofenherdes; ausserdem war auch zwischen den Quarzkörnern unreines derbes Material ausgebildet. Die Krystalle, deren Grösse in der Richtung der grössten Ausdehnung nur 2-3 mm beträgt, sind meistens sehr unvollstïndig entwickelt, so dass an jedem Individuum nur einige Flächen $\mathrm{zu}$ beobachten sind. Im allgerneinen IIabitus stimmen sie mit den von Scacchi beschriebenen gut überein und haben wie diese eine flächenreiche Hauptzone längs der Symmetrieaxe des monoklinen Systems.

Die Winkelmessungen haben ein nicht besonders gutes lesultat gelicfert; die Nlïchen gaben im Allgemeinen eine Mehrzahl von Reflexen. Infolgedessen ist, wie auch aus den Controlwinkeln hervorgeht, dem berechneten $\Lambda$ xenverhältnisse eine grössere Genauigkeit nicht zuzuschreiben. Da aber dieses Axenverhältniss von dem Scacchi'schen nicht unbeträchtlich abweicht und dieser Forscher gar keine Controle über seine Messungen mittheilt, wird dasselbe hier des Vergleiches wegen angeführt. Die Aufstellung ist die von Goldschmidt zuletzt vorgeschlagene, die Flïchenbezeichnungen sind aber die von Scacchi eingeführten.

Krystallsystem: Monoklin.

$$
\begin{gathered}
a: b: c=1,323: 1: 1,203 ; \beta=108^{0} 31^{\prime} . \\
(a: b: c=1,3042: 1: 1,2100 \\
\left.\beta=108^{0} 14^{\prime} \text { nach Scacchi }\right) .
\end{gathered}
$$

Folgende Formen sind beobachtet: $g=\{100\}, C=\{010\}, d=\{001\}, \mu=$ $\{701\}, e=\{101\}, B=\{103\}, A=\{101\}$, $h=\{\bar{T} .0 .3\}, s=\{011\}, \omega=\{\bar{T} 11\}$ und Fig. 1.

$r=\left\{T_{12}\right\}$. S. beistehende Figur, welche mit einer stärkeren Drehung als

1) Atti della R. Accademia delle sicienze Fisiche e Matematiche di Napoli 1873.

2) Krystallformen der Mineralien 1886.

3) Krystallformen der Mineralien 1890.

4) Krystallographische Winkeltabellen 1897. 
gewöhnlich gezeichnet ist, um die lilichen besser hervortreten zu lassen. Neu sind das Orthodoma,$\iota=\{701\}$ und die Hemipyramide $\omega=\{\overline{1} \mid 1\}$.

\begin{tabular}{|c|c|c|c|}
\hline & Gemessen: & Bcrechnet: & Nach Scacchi \\
\hline$d: g=(001):(100)$ & $71029^{\prime}$ & - & $71045^{\prime}$ \\
\hline$d: \iota=(001):(701)$ & 6330 & $63^{0} 25^{\prime}$ & - \\
\hline$d: e=(001):(101)$ & $34 \quad 2$ & 3347 & $34 \quad 19$ \\
\hline$d: B=(001):(103)$ & 1537 & $14 \quad 42$ & 14. 59 \\
\hline$d: A=(001):(T 01)$ & 5028 & - & $\ddot{519} 9$ \\
\hline$d: h=(001):\left(T_{T} .0 .3\right)$ & 9038 & 913 & 91 \\
\hline$d: s=(001):(011)$ & $48 \quad 45$ & - & - \\
\hline$g:(j=(100):(111)$ & 11141 & 11145 & - \\
\hline$A: s=(\overline{1} 01):(011)$ & $64 \ddot{30}$ & $6 \breve{11}$ & 6541 \\
\hline$A: r=(T 01):(T 12)$ & 3736 & 387 & 3816 \\
\hline
\end{tabular}

Die hier als gemessene angegebenen Winkel sind die Mittelwerthe von Beobachtungen an sechs verschiedenen Krystallen.

Die ganze Reihe der am Dolerophanit beobachteten Formen wird somit:

$$
\begin{array}{llllc}
g=\{100\} & t=\{110\} & e=\{101\} & q=\{112\} & n=\{\overline{2} 33\} \\
C=\{010\} & s=\{011\} & B=\{103\} & \omega=\{111\} & \tau(?)=\{1 T .8 .3\} \\
d=\{001\} & \iota=\{701\} & A=\{101\} & p=\{114\} & m(?)=\{\overline{7} 69\} \\
& f=\{301\} & h=\{1 T .0 .3\} & r=\{112\} &
\end{array}
$$

Die zwei zuletzt aufgezählten Pyramiden werden von Goldschmidt als fraglich betrachtet. An den von Scacchi beschriebenen Krystallen scheinen - nach der Figur zu schliessen - die Formen $B, d$ und $A$ vorwaltend $\mathrm{zu}$ sein. An den künstlichen Krystallen von ftvidaberg sind in der IIauptzone $g, e, d$ und $A$ im Allgemeinen mit grösseren Flächen ausgebildet, während die anderen Formen der nämlichen Zone als Kantenabstumpfungen, und zuweilen als sehr schmale, auftreten. Die Zahl der terminalen Formen ist an diesen Krystallen viel geringer als an jenen.

Die Kenntniss des IDolerophanit konnte durch eine Untersuchung über die physikalischen Eigenschaften erweitert werden, welche Untersuchung jedoch in Bezug auf die Stärke der Lichtbrechung und die Grüsse des specifischen Gewichtes unvollständig bleiben musste. Erstens ist nämlich die Iichtbrechung höher als diejenige der Flüssigkeiten, welche bei der Messung mit 'Totalreflectometer benutzt werden, zweitens gestattet die Grösse des specifischen Gewichtes eine Verwendung der Schwebemethode, und die Schwierigkeit, eine ausreichende Menge reines Material zu isoliren, eine Verwendung der Pyknometermethode nicht.

Spaltbarkeit sehr vollkommen nach $\{001\}$. J)ie optischen Axen liegen in der Symmetrieebene, und die geneigte Dispersion ist sehr deutlich. Eine Mittellinie sleht fast senkrecht $\mathrm{zu}\{001\}$, und durch diese liiiche gemessen ist der Axonwinkel in Glas ca. 850; $\varrho>u^{\prime}$. Der Pleochroïsmus der Spalt- 
platten parallel der Basis sehr deutlich, und zwar ist die Kürperfarbe für Schwingungen

parallel der $b$ - $A x e$ dunkel orangegelb bis rothbraun, in der symmetrieebene citronengelb bis dunkel braungelb,

je nach der kleineren oder grüsseren Dicke der Platte.

Um auf die erwähnte Isomorphiefrage zurückzukommen, kann jetzt constatirt werden, dass eine Isomorphie, wie sie Goldschmidt 18861890 gemeint hat, nicht müglich ist, denn wenn dic betreffenden Mineralien übcrhaupt isomorph sind, so müssen doch die Flüchen der vollkommenen Spaltbarkeit (bei Lanarkit ${ }^{1}$ ) die lläche $c$ und bei Dolerophanit die Flïche $d$ ) einander entsprechen. Dies ist aber nach der oben erwähnten Analogisirung nicht der Fall. Nach dieser ist nümlich für Lanarkit:

$$
\begin{aligned}
& a=\{100\} ; \quad a: \mu=180^{\circ}-\beta=60037^{\prime} \\
& \mu=\{001\} ; \quad \mu: c=273 ; \\
& C=\{102\} ;
\end{aligned}
$$

und für Dolerophanit:

$$
\begin{aligned}
& g=\{100\} ; \quad g: A=180^{0}-\beta=57^{0} 6^{\prime} \text { nach Scacchi } \\
& A=\{001\} ; \quad A: d=\quad=319- \\
& d=\{\overline{101}\}
\end{aligned}
$$

Die Spaltllächen liegen somit bei beiden Mineralien im hinteren Winkel $\not$, erhalten aber verschiedene Symbole und künnen infolgedessen einander nicht entsprechen. Darum kann die gesuchte Isomorphie sich nicht auf diese Weise ergeben.

Besser wäre dann vielleicht für Dolerophanit die spätere Aufstellung Goldschmidt's, die auch hier im Vorigen benutzt worden ist, zu adoptiren und für Lanarkit eine ihr entsprechende zu suchen. Fine solche ergiebt sich auch auf folgende Weise.

Fig. 2.

Dolerophanil.

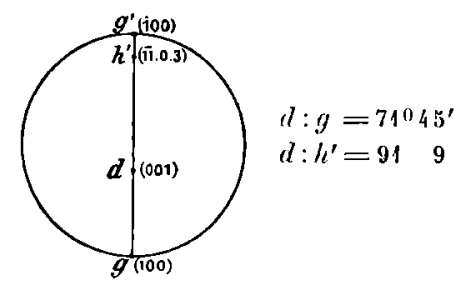

Fig. 3.

Lanarkit.

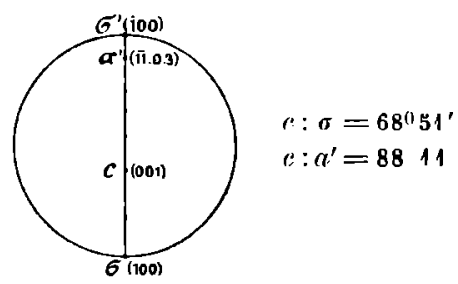

1) Hier und im folgenden sind fïr Lanarkit die Constanten und lifächenbezeichnungen von Schraul benutzt worden: $\Lambda$. Schraul, Die krystallographisithen Conslanten des Lanarkit, diese \%eilschr. 1877, 1, 31. 
Bei dieser Transformation sind für Lanarkit die neuen Indices $\{s t u\}$ $(t=0)$ der Formen der Symmetriezone aus den Schrauf'schen Indices $\{h k l\}(k=0)$ durch folgende Formel zu finden:

$$
s: u=-22 h: 3(2 h+3 l) \text {. }
$$

Somil werden für:

\begin{tabular}{|c|c|c|}
\hline & $=\{30 \overline{2}\}$ & $\{s u t\}=\{100\}$ \\
\hline & $=\{001\}$ & $\{001\}$ \\
\hline & $=\{100\}$ & $\{\bar{T} \bar{T} .0 .3\}$ \\
\hline & $=\{103\}$ & $\{\overline{2} 03\}$ \\
\hline & $=\{131\}$ & $\{\overline{2} \overline{2} . t .15\}$ \\
\hline & $=\{1.10 .5\}$ & $\{\overline{2} \overline{2} \cdot t .51\}$ \\
\hline
\end{tabular}

Diese Aufstellung, die für Dolerophanit eine ganz natürliche ist, wird für Lanarkit eine auf̣'s Aeusserste geschranbte und kann für letzteren nicht adoptirt werden.

Es können also die beiden Mineralien Dolerophanit und Lanarkit als wahrscheinlich mil einander nicht isomorph betrachtet werden; die behauptete Isomorphie beschränkt sich somit auf eine zufällige Aehnlichkeit der Winkel, eine Aehnlichkeit, für welche - des grossen Flächenreichthums des Dolerophanits zufolge -- eine ziemliche Wahrscheinlichkeit vorhanden sein muss. Diese Deutung wird auch durch die Verschiedenheit der optischen Eigenschaften der betreflenden Mineralien bestätigt. Bei Dolerophanit steht eine Mittellinie fast senkrecht zur Fläche der vollkommenen Spaltbarkeit, bei Lanarkit ist die Lage der Mittellinien eine andere ${ }^{1}$ ).

Wenn also die krystallographischen und physikalischen Verhältnisse des Dolerophanits und des Lanarkits dafür sprechen, dass eine Isomorphie hier nicht existirt, so ist dieses als etwas Eigenthümliches nicht zu betrachten; die Verbindungen von zweiwerthigem Kupfer und Blei sind ja auch sonst mit einander nicht isomorph. In den von Arzruni ${ }^{2}$ ) aufgestellten »isomorphen Reihen der Elemente " kommen $\stackrel{\mathrm{II}}{\mathrm{C} u}$ und $\stackrel{\mathrm{II}}{\mathrm{Pb}}$ nicht in den gleichen Reihen vor. $\stackrel{\text { II }}{P b}$ gehört der leihe: $C a, S r, B a, P b$ und $C u$ der Reihe: $M g, \stackrel{\text { II }}{M n}, \stackrel{\mathrm{II}}{\mathrm{F}}, \stackrel{\mathrm{II}}{\mathrm{N}} i, \stackrel{\mathrm{II}}{\mathrm{C}}, \mathrm{Zn}, \stackrel{\mathrm{II}}{\mathrm{C}} u$ etc. an.

Jedenfalls ist jedoch für die schliessliche Entscheidung der Frage eine genauere Kenntniss der. Formenreihe des Lanarkits erforderlich.

1) Jannettaz, Note sur un Sulfate bibasique de plomb de l'Ariège.

2) Beziehungen zwischen Krystallform und chemischer Zusammensetzung. Braunschweig 1893. In der *Theoretischen Chemie von Nernst, dritte Aufl., S. 179. 